\title{
The Use of Measurement Systems to Support Patient Self-Management of Long-Term Conditions: An Overview of Opportunities and Challenges
}

This article was published in the following Dove Press journal:

Patient Related Outcome Measures

\author{
Michelle $M$ Holmes $\mathbb{D}^{1,2}$ \\ Sabina Stanescu ${ }^{2}$ \\ Felicity L Bishop (D) ${ }^{2}$ \\ 'AECC University College, \\ Bournemouth, Dorset, UK; ${ }^{2}$ Department \\ of Psychology, University of \\ Southampton, Southampton, \\ Hampshire, UK
}

\begin{abstract}
Long-term conditions are a major public health concern that present many challenges for patients living with them. There is increasing policy focus on promoting patient self-management and supporting patients to take ownership of managing their conditions. Because long term conditions often fluctuate over time, ongoing monitoring of disease activity is necessary for self-management; this can be achieved through using Patient Reported Outcome Measures (PROMs). PROMs can provide additional information about patients' symptoms, functioning, and emotional wellbeing, informing clinical care for patients. Measurement systems are an innovative method to gather and report PROMs throughout a patient's course of care, to support clinical practice and improve overall quality of care. Measurement systems are often delivered via a digital platform, which can convey patient-reported information to healthcare professionals and provide tailored selfmanagement advice to patients, all based on information collected via PROMs. There are a number of potential benefits of this approach to self-management. Measurement systems can improve clinical practice, creating efficient clinical encounters and positively influencing patient-clinician interactions. The use of monitoring throughout a patient's care is also thought to empower patients, by improving their knowledge of their condition, increasing their engagement with their health, and influencing their overall management of their condition. Challenges associated with using measurement systems in this way include finding appropriate PROMs, provisioning of suitable technology, and limiting the burden for patients. To increase the implementation of measurement systems into practice it is important to consider how to engage and educate healthcare professionals and patients to empower their use. Overall, adopting measurement systems into clinical practice may improve clinicians' ability to support patient self-management of long-term conditions.
\end{abstract}

Keywords: chronic disease, patient outcome assessment, patient reported outcome measures, self-management

\section{Introduction}

With the advances in medicine seen throughout the 20th and early 21 st centuries, previously fatal conditions are now being managed for lengthy time periods, resulting in large numbers of people living with long-term conditions. Long-term conditions are defined as "a condition that cannot, at present, be cured but controlled by medication and/or other treatment/therapies". 1 There is an increasing prevalence of long-term conditions in the UK: over fifteen million people in England have a long-term condition and this is projected to rise to 18 million by
Correspondence: Michelle M Holmes AECC University College, Bournemouth, Dorset, UK

Tel +44I202436252

Email mholmes@aecc.ac.uk 
2025. ${ }^{2}$ According to the World Health Organisation (WHO), long term conditions such as heart disease, stroke, cancer, diabetes and chronic lung disease are responsible for almost $70 \%$ of deaths worldwide, $80 \%$ of which could have been prevented if management strategies were effective. $^{3}$

Recently, there has been a paradigm shift in health care, from an illness-focused approached, to a personcentred approach; within this paradigm, one important aim is to achieve better quality of life for individuals with long-term conditions. As well as commissioning services and providing care, policy makers are encouraging patients to take an active role in managing their health. The person-centred approach advocates patient selfmanagement, which refers to any action taken by people to recognise, treat and manage their own health or chronic conditions, either independently or in conjunction with the healthcare system. ${ }^{4}$ In this context, self-management of chronic illness involves patients recognising their health needs and performing health promotion activities, activating healthcare resources, obtaining support, as well as ongoing processing and adjustment to living with a chronic illness. ${ }^{5,6}$ Related to self-management, taking ownership of one's health is crucial to achieving positive outcomes and better quality of life. ${ }^{7}$ However, selfmanagement is not straight forward and can be challenging for many patients. It is therefore important to develop methods to support and empower patients to self-manage.

Patient reported outcome measures (PROMs) and their implementation within measurement systems constitute one promising approach to supporting patient self-management of long-term conditions. PROMs are standardized instruments for collecting patients' perceptions of and views about their health. ${ }^{8,9}$ PROMs can capture valuable information on the outcomes that are meaningful to patients, including symptoms, functioning, and emotional wellbeing ${ }^{10}$ as well as capturing patients' broader perspectives on their health; they often use continuous measures that permit nuance and subjectivity. ${ }^{11}$ They can be used by clinicians to inform clinical care, extending clinical knowledge beyond test results (which can be poorly correlated with clinical status and quality-of-life) ${ }^{12}$ and encouraging clinicians to attend to issues that are important to patients such as treatment burden and function. ${ }^{13}$ Measurement systems are an innovative digital method to gather and report PROMs throughout a patient's course of care, conveying patient-reported information to healthcare professionals and provide tailored self-management advice to patients based on information collected via PROMs.

Historically self-management interventions have been delivered in person by healthcare professionals. ${ }^{4}$ However, measurement systems are often delivered via electronic platforms, opening up opportunities for other modes of encouraging and facilitating self-management. And PROMs can be implemented through these measurement systems, facilitating patient self-monitoring of their own health and disease activity, prompting them to reflect and act upon their own data and providing this information to healthcare professionals. ${ }^{14}$ When patients have access to their own data, they can adjust their self-management activities accordingly. Additionally, these systems have the ability to provide tailored self-management advice based on information provided by patients via PROMs. ${ }^{15,16}$

For patients with long-term conditions, the need for ongoing self-monitoring is particularly salient. Furthermore, the care and follow-up pathways, as well as the relationships with healthcare professionals may be lifelong. Well-designed measurement systems therefore have great potential to help support clinical care and self-management in this population. These systems help by gathering PROMs data not just before and after treatment, but also over the longer term throughout the course of ongoing clinical and self-management. Measurement systems thus offer a strategic long-term approach to monitoring conditions using PROMs to support clinical practice and improve the overall quality of care. ${ }^{17}$

In this article we will discuss the use of measurement systems for self-management of long-term conditions. The present article outlines theoretical underpinnings and development of using measurement systems and the benefits to clinical practice and patients with long-term conditions. We will also consider the current challenges to the use of these systems and describe facilitators to successful implementation.

\section{Theoretical Perspectives}

In the development of any self-management intervention it is essential to consider the theoretical basis explaining how intervention components may elicit positive effects. The theoretical framework underpinning measurement systems for chronic conditions is currently underdeveloped, with theoretical explanations often focusing explicitly on PROMs. ${ }^{18}$ Additionally, most measurement systems have taken a single-illness approach, i.e., they have focused on monitoring the parameters of a single specific health condition, making it difficult to ascertain an overarching 
approach that would be appropriate across diverse chronic conditions and multimorbidity. However, in studies with an explicit theoretical basis some theories are applicable across multiple measurement systems.

There is a focus in modern medicine to move away from medical decisions based on clinical experience and a "diseasecentred" approach, to a personalised, "patient-centred" focus. Patient-centered healthcare is a multidimensional construct, that encompasses viewing the patient as a whole person, sharing power and responsibility, and mutual participation in the consultation and decision-making. ${ }^{19}$ Patient-centered care is also thought to be associated with the use of measurement systems and PROMs, which may enhance patient-centered care. ${ }^{10,20,21}$ Indeed, ongoing monitoring through the use of appropriately-broad and patient-centered PROMs (encompassing health-related factors important to the specific patient population rather than having a narrow focus on symptoms) could encourage clinicians to look at the whole person, to focus on each individual's experience of illness and to engage in shared decision-making with patients. In other words, it could help move clinicians towards preventive, personalised and participatory medicine, provided patients are appropriately supported to take on a more active role in selfmanagement. $^{22}$

Self-management is rooted in self-efficacy theory, where self-efficacy is the extent to which a person believes in their ability to perform a certain behaviour. ${ }^{23}$ According to self-efficacy theory, these beliefs can influence individuals' choices of action, the effort they make to complete tasks, their perseverance and resilience in the face of setbacks, and their experience of stress. ${ }^{24}$ Individuals with high self-efficacy are more likely to master problems, and to recover from setbacks; as such they find it easier to learn self-management strategies and adhere to them. Patients self-monitoring and receiving information on their health feel they have the appropriate knowledge, skills, and resources to self-manage their condition, which has the potential to improve their self-efficacy for undertaking self-management behaviour. ${ }^{20,25}$

Self-management is complex and very often undertaken in conjunction with lifestyle choices and in the context of particular relationship dynamics. The Individual and Family Selfmanagement Theory considers both the individual process and wider context when describing the drive for employing selfmanagement strategies. ${ }^{26}$ As part of the self-management process, patients need the self-regulation skills of goal setting, self-monitoring, decision-making, and reflective thinking. Measurement systems provide opportunities for self- monitoring and reflective thinking and may be important components of self-management interventions conceptualised in these terms. ${ }^{27}$

Health locus of control refers to the patient's beliefs about whether the control of health issues derives from external influences or is vested internally by the patient themselves. These beliefs are thought to be based on patients' past experience of health. ${ }^{28}$ For effective selfmanagement, internal locus of control needs to be encouraged; i.e., patients believe that they themselves have some control over the management and monitoring of their conditions, rather than believing that only others, such as healthcare professionals, have this control (termed external locus of control). ${ }^{29}$ Measurement systems provide an opportunity for patients to reinforce internal locus of control as it enables them to be more empowered and engaged in self-managing their health.

Finally, a more recent concept, patient activation, has become important in the UK setting. Patient activation refers to knowledge, skills and confidence a person has in managing their own health or condition. ${ }^{30}$ Patient activation and encouraging self-management have been included in the newly published National Health Service (NHS) Long-Term Plan. ${ }^{31}$ Measurement systems combine patient activation and engagement by providing feedback and potentially tailored information from self-monitoring to patients that enables them to actively participate in the management of their conditions.

\section{The Development of Electronic Measurement Systems}

Whilst existing approaches to PROMs have typically focused on paper-based systems, these can be limiting and inefficient in clinical practice, ${ }^{32,33}$ burdensome for patients, and subject to missing data. ${ }^{15}$ Advances in technology have facilitated the development of electronic systems to measure patients' health. There has been a rise in the use of physiological monitoring tools, including wearable activity trackers. $^{34,35}$ Electronic systems also allow PROMs to be pragmatically collected, stored and reported in routine clinical practice. A decade ago, researchers were excited by the increasing availability of electronic tools and measurement systems to collect PROMs. ${ }^{33}$ Now electronic methods for collecting PROMs can include: personal computers, webbased systems, telephone, mobile apps, online diaries, and email reminders. ${ }^{33,36-38}$ A review of electronic PROM systems in cancer care identified 33 systems, with most (63\%) 
being used throughout treatment and many (40\%) for follow-up care. ${ }^{37}$

Much of the literature around the use of electronic systems to record PROMs has focused on the adaptation of paper PROMs to equivalent electronic PROMs (ePROMs). To retain the same psychometric properties (i.e., validity and reliability) and to be used interchangeably if necessary, ePROMs must have equivalent psychometric properties has the original paper-based questionnaires. ${ }^{39}$ A Cochrane review found consistency between surveys collected using mobile apps and their paper equivalents. ${ }^{40}$ The results suggest that the psychometric properties were not compromised when data were collected via apps compared to on paper forms. For example, the Sickle cell disease Mobile Application to Record symptoms via Technology (SMART), is a PROM for patients to monitor their pain that has demonstrated equivalence to a paperbased version of the tool. ${ }^{15}$

In addition to reducing patient burden, there are several benefits for moving towards electronic instead of paper-based measurement systems. Data processing is more efficient and less error-prone, as data can be scored automatically without requiring data input or transfer between systems. ${ }^{36,41}$ Data capture can also be more efficient and comprehensive, as PROMs can be readily completed in between clinic visits with minimal additional resources. For patients with rheumatoid arthritis completing self-assessment questionnaires via a website, this automated scoring and continual data capture outside of clinic visits were found to be beneficial in routine clinical practice. $^{42}$ Patients also prefer using electronic systems to paper, regardless of age, sex, race, or educational level. $^{43}$

One of the main advantages of electronic systems is the increased access to monitoring that they can afford. Electronic systems mean patients can complete measurements at home when convenient for them. A concern with their implementation in clinical practice is patients having difficulties with accessing and navigating electronic systems. However, in Great Britain it is estimated that $90 \%$ of households have internet access, with $73 \%$ of adults accessing the internet using a mobile phone. ${ }^{44}$ The use of mobile apps and web-based systems were previously thought to exclude elderly populations, who are more likely to have (multiple) long-term conditions, and are less likely to use the internet. Recently this does not seem to be the case, with older adults found to be willing and quick to learn to incorporate mobile and digital interventions into the self-management of their conditions. ${ }^{45}$

\section{Improvements in Clinical Practice Efficient Clinical Processes}

Electronic measurement systems may be a cost-effective intervention to aid self-management of chronic conditions. Patient health status can be monitored remotely, and healthcare professionals can regularly collect data at low cost. This information provided outside of patient-clinician interactions makes monitoring patients less burdensome for healthcare professionals during the clinical encounter aiming to reduce the burden on strained healthcare services and professionals. ${ }^{41,46}$ Additionally, monitoring patients' health outside of the clinical encounter is thought to streamline visits to healthcare professionals. ${ }^{47}$

Measurement systems may also be fully integrated into clinical practice and even replace unnecessary visits to healthcare professionals. An example is the AmbuFlex, a web-based measurement system for patients with chronic or malignant conditions. ${ }^{17}$ This system makes automated decisions in which the information provided by patients through PROMs identifies patients who need to attention from a healthcare professional. Systems integrated in this way may reduce the treatment burden with patients requiring fewer visits to healthcare professionals for follow-ups with their condition. ${ }^{48}$

Systems can also be used to monitor changes in patients' symptoms with the opportunity for healthcare professionals to then intervene, request to see a patient, and modify treatment. One example of this, is patients undergoing treatment for head and neck cancer to record symptoms and side-effects of treatment via a mobile app. ${ }^{49}$ This approach is thought to be a potentially effective way to avoid the progression of symptoms, delay in diagnosis, undertreatment, and hospital readmission. If measurement systems are used routinely, this may reduce unnecessary visits and reduce the burden on healthcare services.

\section{Patient-Clinician Interaction}

Integrating technology and PROMs through measurement systems has the potential to improve healthcare services by influencing patient-clinician interactions. ${ }^{46}$ Previous frameworks have been developed to understand the theory behind the use of PROMs in clinical practice. Santana and Feeny (2014) propose that PROMs have a cascading effect, with completion of PROMs eventually leading to improvements in outcomes. ${ }^{50}$ The authors theorized that completing PROMs may influence communication, raising patient awareness of symptoms and facilitating patient communication of 
symptoms to clinicians, as well as encouraging clinicians to discuss issues picked up by the PROMs that might not otherwise have been raised in consultations. PROMs can change clinicians understanding of their patients' needs and expand their focus to a wide range of issues related to a patients' condition and symptoms. ${ }^{51}$ Patients feel that if clinicians read the information they have provided, they will gain a deeper understanding of their experience with their condition. ${ }^{36}$ Healthcare professionals have better insight into the disease activity and identification of issues that may have previously gone undetected. $^{48}$ For example, healthcare professionals using a mobile-based PROM system for patients with complex needs reported that PROMs provided them with additional information on patients' wellbeing. ${ }^{52}$

The use of PROMs is thought to improve patientclinician interaction by promoting clear communication with healthcare professionals and as a prompt for conversation. $^{36}$ Patients feel that this data helps focus healthcare professionals on the problems that are important to them. ${ }^{47}$ In interviews with consumers of a mental health service and their carers, participants believed that the differences in perceived health status and clinicians views could be used as a prompt to discuss issues. ${ }^{51}$ Daily self-reporting was associated with increased contribution in follow-up consultations by initiating conversations and linking hypertension to lifestyle variables. ${ }^{53}$

Studies investigating the use of PROMs in routine clinical practice have reported improvement in the diagnosis of conditions, patient-clinician communication, and shared-decision-making. ${ }^{18,54}$ In a review of qualitative research on clinicians' experiences of using PROMs, clinicians thought PROMs can impact on processes of care, such as communication, shared decision-making and care planning. ${ }^{55}$ Evaluations of PROMs in routine clinical practice have reported improvements in patient-clinician communication in a variety of settings including oncology ${ }^{56}$ and patients with complex healthcare needs. ${ }^{52}$

\section{Developing Empowerment and Engagement in Patients Patient Knowledge and Empowerment}

Measurement systems may improve patient knowledge of their condition. Through provision of information and increased involvement in clinical encounters, patients may be empowered to self-manage their condition. ${ }^{33,57}$ For example, patients with COPD felt the monitoring of conditions through a telehealth system improved their knowledge of their condition. ${ }^{38}$ Patients felt the clinical data provided was beneficial, and helped them to feel reassured, supported, and empowered. Likewise, other measurement systems are designed to provide clinical data to patients with the aim of monitoring their condition. The Living with Lymphoma Intervention, a web-based self-management intervention, provides a graphic overview of symptom trajectory and functioning score as well as an option to compare scores to other patients, which helps to reassure patients of their experiences and empowers them to take an active role in managing their condition. ${ }^{58}$

This access to information and data from measurement systems improves patient perceived control over health. ${ }^{59}$ After the implementation of a web-based tool allowing adolescents to self-report their pain, participants in one study reported that creating their own pain record improved their perceived control over and ownership of their pain. ${ }^{36}$ Similarly, in a telehealth service for patients with long-term conditions, patients reported increased confidence in dealing with symptoms and greater independence. ${ }^{60}$

Measurement systems that provide self-management advice on the basis of PROM data were seen by healthcare professionals as helping patients feel more empowered. ${ }^{14}$ This information can be acted upon immediately, without visiting a healthcare professional, thus improving patients' perceived and potentially actual control of their condition. For example, an e-Health application developed for cancer survivors monitored quality of life through PROMs. Patients received personalised feedback automatically, including advice, supportive care options and information about seeking health services. This increased patient activation, allowing patients to take control of their health and adopt an active role in managing their symptoms. ${ }^{61}$

\section{Patient Engagement in Self-Management}

Measurement systems provide opportunities for patients to self-manage their health and encourage them to actively participate in the management of their condition. ${ }^{62}$ The process of implementing measurement systems into routine clinical practice is thought to prompt conversation between patients and clinicians, aiming for patients to more actively engage in the care of their condition. ${ }^{56}$ The data from selfmonitoring health and symptoms may start a dialogue about care and treatment options, with patients feeling empowered in the decision-making process.

Goal-setting is an important part of clinical management, helping patients to prioritize their health, coordinate care plans and support treatment from healthcare professionals. ${ }^{63}$ Often 
health goals are not discussed in the patient-clinician encounter due to a lack of time. ${ }^{64}$ PROMs bring awareness of patient's desired outcomes and treatment goal to the clinician, which can prompt discussion of the patient's expectations and realistic goal setting. Clinician and patient perspectives can be integrated to develop mutually acceptable treatment and health goals. $^{65}$

By improving patient knowledge and involvement in goal setting and self-monitoring, patients may feel more engaged in their care and be more adherent to agreed upon self-care actions. ${ }^{48}$ Additionally, patients may take more control over managing their health. Patients with lung cancer using a measurement system, felt that the system helped them to manage their symptoms, reduced uncertainty regarding their condition, and assured patients when to contact their healthcare team. ${ }^{14}$ Similarly, in a study of telehealth monitoring for patients with COPD, most patients said they felt more involved in their care and more able to manage their own care during the telehealth pilot. $^{60}$ The continued monitoring of their condition and reporting of changes due to any treatment or self-care activities, can influence patients' adherence to and engagement with ongoing treatment. ${ }^{66}$ A randomized-controlled trial of the integration of PROMs and patient education for patients with rheumatoid arthritis found reduced disease activity and improved adherence to medication. ${ }^{11}$

\section{Challenges of Measurement Systems}

\section{Acceptability and Applicability of PROMs}

The implementation of self-monitoring for self-management in routine clinical practice must consider the appropriate measurement tools. PROMs have a variety of purposes, such as in healthcare evaluation and examining treatment effectiveness, as well as with individual patients. In designing measurement systems, it is important to consider the goals of self-monitoring and assess the patients' needs. Best practice would be to involve all stakeholders, (patients, clinicians, management) in the choice of outcomes to be measured, ${ }^{51}$ such as symptoms, activity limitations, or quality of life. ${ }^{21}$ In particular, patients should be involved in this process to ensure that the measurement system is patientcentred and not intrusive. ${ }^{61}$ The measurement must also be clinically relevant and applicable to routine clinical practice for clinicians to engage with the systems. ${ }^{18,56,67}$

Introducing PROMs into healthcare systems requires identifying suitable measures of agreed upon outcomes for routine clinical practice. ${ }^{21}$ Appropriate PROMs are supported by research demonstrating their acceptability to patients, reliability, validity, and responsiveness. ${ }^{9}$ Additionally, PROMs created on a paper format must be adapted into an electronic medium ensuring the retention of measurement properties. ${ }^{37}$ With PROMs developed for a number of purposes and for different contexts, it is a challenge to identify the appropriate PROM. $^{8}$ A range of PROMs may be required into convey patients' experiences with their condition. ${ }^{51}$

An additional challenge is to identify the optimal timing of measurements and the frequency of asking patients to complete PROMs. ${ }^{41}$ Intensive assessments may be useful for clinicians, but this must be considered against the potential burden for patients. ${ }^{56}$ Survey length and complexity of questionnaires were identified as barriers for implementation of self-monitoring, suggesting PROMs should be brief and multidimensional. ${ }^{47}$ As well as minimising patient burden this reduces the time required for healthcare professionals to review the patient-completed data. ${ }^{47}$

\section{Technology}

Logistical difficulties and technological constraints have deterred the use of measurement systems in clinical practice. ${ }^{46}$ Health services require the appropriate hardware, internet access, and software to use the electronic system. ${ }^{36}$ The system must also be developed in a manner which is conducive to use within clinical settings. Healthcare professionals feel that unhelpful presentation of information may limit how effective measurement systems are in practice. $^{52}$ Therefore, PROMs data must be presented in a format that is helpful to the clinician, which may include both tabular and graphical formats of PROMs as well as numerical text formats. ${ }^{56}$

Patients need computer facilities and access to the internet in order to engage with measurement systems. Additionally, systems must be designed to produce data in a format that is easily accessible for patients to interpret. $^{48}$ In a feasibility study of online PROMs for rheumatoid arthritis patients, some patients refused to participate due to their inexperience with a computer or the internet. ${ }^{57}$ Additionally, $74 \%$ of participants (58) required assistance with the system. Although patients were willing to use the system, fewer than half continued use in the six months after registration. Similarly, patients with multiple sclerosis accessing a web-based PROMs system, found that $12.9 \%$ of subjects had difficulties with initial access to the system, with then $22.6 \%$ later having difficulty. ${ }^{59}$ 
Overall however, electronic systems for PROMs are thought to be broadly acceptable to patients. ${ }^{56}$ In a study to assess the feasibility and acceptability of PROMs with patients with rheumatoid arthritis, patients' perceived a web-based PROM as easy, and reported willingness to fill in the questionnaires at home. ${ }^{57}$ For multiple sclerosis patients completing monthly PROMs via an online selfassessment tool, the patient burden was low. ${ }^{59}$ Patients with rheumatoid arthritis were also engaged with the use of an electronic system, finding the technology ease to use. ${ }^{42}$ Despite concerns that older patients will have difficulties in using technology, studies report no trends between difficulties using systems and age, with older patients able to use systems. ${ }^{42,59}$

\section{Stakeholder Concerns}

Measurements systems are intended to improve patient care, quality of health service provision, and enhance patient self-management. Therefore, clinicians and healthcare providers may choose to implement PROMs for all patients, as part of routine clinical practice. ${ }^{68}$ This strategy of implementation may raise patient concerns about the use of their data and may see this as an invasion of privacy. However, if healthcare organizations treat this routine data collection according to strict legal and governance principles for research, this may significantly reduce patient participation. ${ }^{68}$ Additionally, many measurement systems have the opportunity for individual patient data to be pooled. This aggregated data can be used for audit, to examine the effectiveness, appropriateness, quality and performance of healthcare. ${ }^{69}$ Pooling data, whilst providing additional information for clinicians to improve patient care, has additional requirements. Data must be stored appropriately with safeguards to protect patient information, and patients must provide additional consent for their data to be used for this purpose. ${ }^{68}$

\section{Implementation of Measurement Systems in Clinical Practice}

Measurement systems have the potential to improve patient care when fully incorporated into routine clinical practice. Currently, healthcare professionals view measurement systems as disruptive to clinical practice. Clinicians will be less engaged if they must spend more time or use more resources to deal with measurement systems. ${ }^{17,47}$ Healthcare professionals must view the systems as part of the clinical process. However, current literature has mostly focused on the efficacy of measurement systems and the feasibility of adopting systems into practice with little research focusing on the implementation of measurement systems into real-world settings. ${ }^{16}$

\section{Clinician Training}

Measurement systems are only sustainable if healthcare professionals are willing to integrate them into their routine clinical practice. ${ }^{52}$ Healthcare professionals vary on whether they find PROMs helpful or not. ${ }^{55}$ In a study of pain settings, while most clinicians believed PROMs can contribute to the initial assessment of a patient, they had mixed views about the use of monitoring to track patients' progress. ${ }^{65}$ Healthcare professionals' lack of knowledge is a significant barrier to the use of measurement systems. For successful implementation, clinicians need to be able to interpret data and to understand the potential utility of measurement systems in clinical practice. ${ }^{18}$

A review of measurement systems in cancer care identified a lack of guidelines for clinicians on how to interpret data and identify meaningful changes. ${ }^{37}$ Educating clinicians on the purposes of the systems and benefits of using them is essential for implementation in clinical practice. ${ }^{70}$ However, information provision and implementation of technology alone may not be sufficient to change clinical practice, particularly concerning complex systems. Therefore, training for healthcare professionals should include the benefits of using the system, as well as administration of the measurement system and how PROMs are scored. ${ }^{51,71}$ Training must also incorporate how to explain the system to patients, how to interpret the results, and how to use the information provided including effective approaches to discuss and respond to issues raised by patients. ${ }^{56}$

\section{Patient Engagement}

The implementation of self-monitoring into clinical practice for self-management must consider patient engagement with the measurement system. For patients to make an informed choice about engaging with measurement systems, patients need to understand the purpose of the measurement system, how to use the system, and the value of self-monitoring. ${ }^{57}$ This enables patients to see measurement systems as an acceptable and appropriate part of their care and engage with the process. Healthcare professionals may need to remind patients to use measurement systems and provide encouragement for self-monitoring of their condition. ${ }^{71}$ If healthcare professionals and support staff 
are engaged with the system, this is likely to facilitate patient interest and involvement. ${ }^{67,71}$

To effectively use measurements systems to improve patient-centered care, the data provided by patients must be integrated into routine clinical practice and discussed between patients and clinicians. ${ }^{36,41}$ Discussion of the data improves patients' understanding of the value of the measurement system and its use within the management of their condition. ${ }^{48}$ Additionally, the importance of the measurement system in self-management must be continually addressed as studies show declining completion rates after initial engagement. ${ }^{59}$

Although electronic systems are thought to be feasible and acceptable to patients, there remain concerns surrounding patients' capabilities to self-monitor their health. In a review of allied health professionals' use of routine outcome measurement, clinicians had concerns about patients' ability to complete PROMs, due to the complicated nature of questions, language barriers, and PROMs being confusing. ${ }^{67}$ Healthcare providers also perceive health literacy to be a potential barrier for patients completing PROMs. ${ }^{47}$ It is therefore essential in the development stages, to involve patients in the design of measurement systems to ensure their usability, acceptability, and usefulness in clinical practice. ${ }^{72}$

\section{Conclusion}

This paper has briefly reviewed the use of electronic measurement systems and illustrating their benefit and potential concerns for patients with long-term conditions. Measurement systems can provide a long-term approach to monitoring conditions, by enabling patients to measure their health and disease activity. These systems may have a role in self-management of long-term conditions as well as supporting informed clinical practice and improving the overall quality of care. Measurement systems are often delivered via an electronic platform, potentially improving equity across healthcare delivery. With patients able to monitor their condition over time, patients can adjust their self-management activities accordingly. Additionally, measurement systems have the ability to provide individualised self-management advice based on patients' self-assessment of their condition. Measurement systems can also improve clinical encounters, empowering patients, increasing their engagement with their health, and influencing their overall management of their condition. While we have noted in this review some evidence to support these potential benefits of measurement systems, it is important to acknowledge that this area requires more and more rigorous studies alongside more theoretical development. With little published research on the use of measurement systems, further research is required to understand which healthcare services they should be incorporated into and how this may impact patients. Researchers should examine the clinical benefits of measurement systems, and any adverse events and unanticipated consequences of their use. Similarly, research is needed to devise and test ways to overcome some of the challenges to self-monitoring and facilitate successful implementation into clinical practice. In conclusion, encouraging patients to self-monitor their health throughout the disease trajectory may enhance self-management and improve healthcare professionals' clinical practice, helping both patients and clinicians to realise patient-centred care.

\section{Disclosure}

The authors report no conflicts of interest in this work.

\section{References}

1. Department of Health. Long Term Conditions Compedium of Information. UK; 2012. Available from: https://www.gov.uk/govern ment/publications/long-term-conditions-compendium-of-informationthird-edition. Accessed 10 December 2019

2. House of Commons Health Committee. Managing the care of people with long-term conditions. Second report of Session. Vol 15. 2014.

3. World Health Organization. Global Action Plan for the Prevention and Control of Noncommunicable Diseases 2013-2020. World Health Organization; 2013:9241506237.

4. Barlow J, Wright C, Sheasby J, Turner A, Hainsworth J. Selfmanagement approaches for people with chronic conditions: a review. Patient Educ Couns. 2002;48(2):177-187. doi:10.1016/S0738-3991(02) 00032-0

5. Schulman-Green D, Jaser S, Martin F, et al. Processes of self-management in chronic illness. $J$ Nurs Scholarsh. 2012;44(2):136-144. doi:10.1111/j.1547-5069.2012.01444.x

6. Dwarswaard J, Bakker EJ, van Staa A, Boeije HR. Self-management support from the perspective of patients with a chronic condition: a thematic synthesis of qualitative studies. Health Expect. 2016;19 (2):194-208. doi:10.1111/hex.12346

7. Jonkman NH, Schuurmans MJ, Jaarsma T, Shortridge-Baggett LM, Hoes AW, Trappenburg JC. Self-management interventions: proposal and validation of a new operational definition. $J$ Clin Epidemiol. 2016;80:34-42. doi:10.1016/j.jclinepi.2016.08.001

8. Dawson J, Doll H, Fitzpatrick R, Jenkinson C, Carr AJ. The routine use of patient reported outcome measures in healthcare settings. $B M J$. 2010;340:c186. doi:10.1136/bmj.c186

9. Fitzpatrick RM, Davey C, Buxton MJ, Jones DR. Evaluating patient-based outcome measures for use in clinical trials. Health Technol Assess. 1998;2(14). doi:10.3310/hta2140

10. Greenhalgh J, Long AF, Flynn R. The use of patient reported outcome measures in routine clinical practice: lack of impact or lack of theory? Soc Sci Med. 2005;60(4):833-843. doi:10.1016/j.socscimed.2004.06.022

11. El Miedany Y, El Gaafary M, El Arousy N, Ahmed I, Youssef S, Palmer D. Arthritis education: the integration of patient-reported outcome measures and patient self-management. Clin Exp Rheumatol. 2012;30(6):899-904.

12. Kiltz U, Boonen A, Braun J, Richter JG. Electronic assessment of disease activity and functioning in patients with axial spondyloarthritis: challenges and unmet needs. Clin Exp Rheumatol. 2016;34(Supp1 101):S57-S61. 
13. Hjollund NHI, Larsen LP, Biering K, Johnsen SP, Riiskjær E, Schougaard LM. Use of patient-reported outcome (PRO) measures at group and patient levels: experiences from the generic integrated PRO system, WestChronic. Interact J Med Res. 2014;3(1):e5. doi:10.2196/ ijmr.2885

14. Maguire R, Ream E, Richardson A, et al. Development of a novel remote patient monitoring system: the advanced symptom management system for radiotherapy to improve the symptom experience of patients with lung cancer receiving radiotherapy. Cancer Nurs. 2015;38(2):E37-E47. doi:10.1097/NCC.0000000000000150

15. Jonassaint CR, Shah N, Jonassaint J, De Castro L. Usability and feasibility of an mHealth intervention for monitoring and managing pain symptoms in sickle cell disease: the Sickle Cell Disease Mobile Application to Record Symptoms via Technology (SMART). Hemoglobin. 2015;39 (3):162-168. doi:10.3109/03630269.2015.1025141

16. Jackson BD, Gray K, Knowles SR, De Cruz P. EHealth technologies in inflammatory bowel disease: a systematic review. J Crohn's Colitis. 2016;10(9):1103-1121. doi:10.1093/ecco-jcc/jjw059

17. Schougaard LMV, Larsen LP, Jessen A, et al. AmbuFlex: tele-patientreported outcomes (telePRO) as the basis for follow-up in chronic and malignant diseases. Qual Life Res. 2016;25(3):525-534. doi:10.1007/s11136-015-1207-0

18. Greenhalgh J, Dalkin S, Gooding K, et al. Functionality and feedback: a realist synthesis of the collation, interpretation and utilisation of patient-reported outcome measures data to improve patient care. Health Serv Delivery Res. 2017;5(2)1-280.

19. Mead N, Bower P. Patient-centredness: a conceptual framework and review of the empirical literature. Soc Sci Med. 2000;51 (7):1087-1110. doi:10.1016/S0277-9536(00)00098-8

20. Greenhalgh J. The applications of PROs in clinical practice: what are they, do they work, and why? Qual Life Re. 2009;18(1):115-123. doi:10.1007/s11136-008-9430-6

21. Snyder CF, Aaronson NK, Choucair AK, et al. Implementing patient-reported outcomes assessment in clinical practice: a review of the options and considerations. Qual Life Re. 2012;21 (8):1305-1314. doi:10.1007/s11136-011-0054-x

22. Lejbkowicz I, Caspi O, Miller A. Participatory medicine and patient empowerment towards personalized healthcare in multiple sclerosis. Expert Rev Neurother. 2012;12(3):343-352. doi:10.1586/ern.11.161

23. Bandura A. Human agency in social cognitive theory. Am Psychol. 1989;44(9):1175. doi:10.1037/0003-066X.44.9.1175

24. Bandura A. Organisational applications of social cognitive theory. Austr J Manage. 1988;13(2):275-302. doi:10.1177/031289628801300210

25. Marshall S, Haywood K, Fitzpatrick R. Impact of patient-reported outcome measures on routine practice: a structured review. $J$ Eval Clin Pract. 2006;12(5):559-568. doi:10.1111/jep.2006.12.issue-5

26. Ryan P, Sawin KJ. The individual and family self-management theory: background and perspectives on context, process, and outcomes. Nurs Outlook. 2009;57(4):217-225. e216. doi:10.1016/j.outlook.2008.10.004

27. Yi JY, Kim Y, Cho Y-M, Kim H. Self-management of chronic conditions using mHealth interventions in Korea: a systematic review. Healthc Inform Res. 2018;24(3):187-197. doi:10.4258/hir.2018.24.3.187

28. Wallston KA. Hocus-pocus, the focus isn't strictly on locus: rotter's social learning theory modified for health. Cognit Ther Res. 1992;16 (2):183-199. doi:10.1007/BF01173488

29. Wallston KA, Maides S, Wallston BS. Health-related information seeking as a function of health-related locus of control and health value. $J$ Res Pers. 1976;10(2):215-222. doi:10.1016/0092-6566(76)90074-X

30. Greene J, Hibbard JH. Why does patient activation matter? An examination of the relationships between patient activation and health-related outcomes. J Gen Intern Med. 2012;27(5):520-526. doi:10.1007/s11606-011-1931-2

31. NHS England. The NHS Long Term Plan. UK; 2019. Available from: https://www.longtermplan.nhs.uk/online-version/. Accessed 10 December 2019
32. Segan L, Nanayakkara S, Mak V, Kaye D. Enhancing self-care strategies in heart failure through patient-reported outcome measures. Intern Med J. 2018;48(8):995-998. doi:10.1111/imj.2018.48.issue-8

33. Fortner B. The challenge of electronically captured patient-reported outcomes. J Oncol Pract. 2007;3(3):120. doi:10.1200/JOP.0731503

34. Chiauzzi E, Rodarte C, DasMahapatra P. Patient-centered activity monitoring in the self-management of chronic health conditions. BMC Med. 2015;13(1):77. doi:10.1186/s12916-015-0319-2

35. Choi EH, Seo JY. U-health for management of chronic diseases-physical activity and therapeutic exercise. $J$ Korean Med Assoc. 2009;52(12):1154-1163. doi:10.5124/jkma.2009.52.12.1154

36. Lalloo C, Stinson JN, Brown SC, Campbell F, Isaac L, Henry JL. PainQuILT: assessing clinical feasibility of a web-based tool for the visual self-report of pain in an interdisciplinary pediatric chronic pain clinic. Clin J Pain. 2014;30(11):934-943. doi:10.1097/AJP.0000000000000049

37. Jensen RE, Snyder CF, Abernethy AP, et al. Review of electronic patient-reported outcomes systems used in cancer clinical care. J Oncol Pract. 2013;10(4):e215-e222.

38. Fairbrother P, Pinnock H, Hanley J, et al. Exploring telemonitoring and self-management by patients with chronic obstructive pulmonary disease: a qualitative study embedded in a randomized controlled trial. Patient Educ Couns. 2013;93(3):403-410. doi:10.1016/j. pec.2013.04.003

39. Coons SJ, Gwaltney CJ, Hays RD, et al. Recommendations on evidence needed to support measurement equivalence between electronic and paper-based patient-reported outcome (PRO) measures: ISPOR ePRO good research practices task force report. Value Health. 2009;12(4):419-429. doi:10.1111/j.1524-4733.2008.00470.x

40. Belisario JSM, Jamsek J, Huckvale K, O’Donoghue J, Morrison CP, Car J. Comparison of self-administered survey questionnaire responses collected using mobile apps versus other methods. Cochrane Database Syst Rev. 2015. doi:10.1002/14651858.MR000042.pub2

41. Jongen PJ, Sanders E, Zwanikken C, et al. Adherence to monthly online self-assessments for short-term monitoring: a 1-year study in relapsing-remitting multiple sclerosis patients after start of disease modifying treatment. Patient Prefer Adherence. 2013;7:293. doi:10.2147/PPA.S40173

42. Walker UA, Mueller RB, Jaeger VK, et al. Disease activity dynamics in rheumatoid arthritis: patients' self-assessment of disease activity via WebApp. Rheumatology. 2017;56(10):1707-1712. doi:10.1093/ rheumatology/kex229

43. Bliven BD, Kaufman SE, Spertus JA. Electronic collection of health-related quality of life data: validity, time benefits, and patient preference. Qual Life Res. 2001;10(1):15-21. doi:10.1023/ A:1016740312904

44. Statistica. UK: smartphone ownership by age from 2012-2017. 2017. Accessed 17/05/18, 2018.

45. Kim BY, Lee J. Smart devices for older adults managing chronic disease: a scoping review. JMIR mHealth and uHealth. 2017;5(5): e69. doi:10.2196/mhealth.7141

46. Chang $\mathrm{CH}$. Patient-reported outcomes measurement and management with innovative methodologies and technologies. Qual Life Res. 2007;16(SUPPL. 1):157-166. doi:10.1007/s11136-007-9196-2

47. Philpot LM, Barnes SA, Brown RM, et al. Barriers and benefits to the use of patient-reported outcome measures in routine clinical care: a qualitative study. Am J Med Qual. 2018;33(4):359-364. doi:10.1177/1062860617745986

48. Catarinella FS, Bos WH. Digital health assessment in rheumatology: current and future possibilities. Clin Exp Rheumatol. 2016;34(101):2-4.

49. Falchook AD, Tracton G, Stravers L, et al. Use of mobile device technology to continuously collect patient-reported symptoms during radiation therapy for head and neck cancer: a prospective feasibility study. $A d v$ Radiat Oncol. 2016;1(2):115-121. doi:10.1016/j.adro.2016.02.001

50. Santana M-J, Feeny D. Framework to assess the effects of using patient-reported outcome measures in chronic care management. Qual Life Res. 2014;23(5):1505-1513. doi:10.1007/s11136-013-0596-1 
51. Callaly T. Introducing the routine use of outcomes measurement to mental health services. Aust Health Rev. 2001;24(1):43-50. doi:10.1071/AH010043

52. Hans PK, Gray CS, Gill A, Tiessen J. The provider perspective: investigating the effect of the Electronic Patient-Reported Outcome (ePRO) mobile application and portal on primary care provider workflow. Prim Health Care Res Dev. 2018;19(2):151-164. doi:10.1017/S1463423617000573

53. Bengtsson U, Kjellgren K, Hallberg I, Lundin M, Mäkitalo Å. Patient contributions during primary care consultations for hypertension after self-reporting via a mobile phone self-management support system. Scand J Prim Health Care. 2018;36(1):70-79. doi:10.1080/ 02813432.2018.1426144

54. Espallargues M, Valderas JM, Alonso J. Provision of feedback on perceived health status to health care professionals: a systematic review of its impact. Med Care. 2000;38(2):175-186. doi:10.1097/ 00005650-200002000-00007

55. Boyce MB, Browne JP, Greenhalgh J. The experiences of professionals with using information from patient-reported outcome measures to improve the quality of healthcare: a systematic review of qualitative research. BMJ Qual Saf. 2014;23(6):508-518. doi:10.1136/bmjqs-2013-002524

56. Gilbert A, Sebag-Montefiore D, Davidson S, Velikova G. Use of patient-reported outcomes to measure symptoms and health related quality of life in the clinic. Gynecol Oncol. 2015;136(3):429-439. doi:10.1016/j.ygyno.2014.11.071

57. Koevoets R, de Glas NA, Le Bourlout C, et al. Autonomous online health assessment questionnaire registry in daily clinical practice. Rheumatology. 2013;52(5):883-887. doi:10.1093/rheumatology/kes389

58. Arts LP, van de Poll-franse LV, Van Den Berg SW, et al. Lymphoma InterVEntion (LIVE)-patient-reported outcome feedback and a webbased self-management intervention for patients with lymphoma: study protocol for a randomised controlled trial. Trials. 2017;18 (1):199. doi:10.1186/s13063-017-1943-2

59. Engelhard MM, Patek SD, Sheridan K, Lach JC, Goldman MD. Remotely engaged: lessons from remote monitoring in multiple sclerosis. Int J Med Inform. 2017;100:26-31. doi:10.1016/j.ijmedinf.2017.01.006

60. Paget T, Jones C, Davies M, Evered C, Lewis C. Using home telehealth to empower patients to monitor and manage long term conditions. Nurs Times. 2010;106(45):17-19.

61. Melissant HC, Verdonck-de Leeuw IM, Lissenberg-Witte BI, Konings IR, Cuijpers P, Van Uden-kraan CF. 'Oncokompas', a web-based selfmanagement application to support patient activation and optimal supportive care: a feasibility study among breast cancer survivors. Acta Oncol. 2018;57(7):924-934. doi:10.1080/0284186X.2018.1438654
62. Cook DJ, Manning DM, Holland DE, et al. Patient engagement and reported outcomes in surgical recovery: effectiveness of an e-health platform. J Am Coll Surg. 2013;217(4):648-655. doi:10.1016/j. jamcollsurg.2013.05.003

63. Gray CS, Wodchis WP, Upshur R, et al. Supporting goal-oriented primary health care for seniors with complex care needs using mobile technology: evaluation and implementation of the health system performance research network, Bridgepoint electronic patient reported outcome tool. JMIR Res Protoc. 2016;5(2):e126. doi: $10.2196 /$ resprot. 5756

64. Ekdahl AW, Hellström I, Andersson L, Friedrichsen M. Too complex and time-consuming to fit in! Physicians' experiences of elderly patients and their participation in medical decision making: a grounded theory study. BMJ Open. 2012;2(3):e001063. doi:10.1136/bmjopen-2012-001063

65. Holmes MM, Lewith G, Newell D, Field J, Bishop FL. The impact of patient-reported outcome measures in clinical practice for pain: a systematic review. Qual Life Res. 2017;26(2):245-257. doi:10.1007/ s11136-016-1449-5

66. Johnson C. Outcome measures for research and clinical practice. J Manipulative Physiol Ther. 2008;31(5):329-330. doi:10.1016/j. jmpt.2008.05.001

67. Duncan EA, Murray J. The barriers and facilitators to routine outcome measurement by allied health professionals in practice: a systematic review. BMC Health Serv Res. 2012;12(1):96. doi:10.1186/1472-6963$12-96$

68. Gensheimer SG, Wu AW, Snyder CF. Oh, the places we'll go: patient-reported outcomes and electronic health records. PatientPatient-Centered Outcomes Res. 2018;11(6):591-598. doi:10.1007/ s40271-018-0321-9

69. Appleby J, Devlin NJ. Measuring NHS Success: Can Patients'Views on Health Outcomes Help to Manage Performance? London: King's Fund; 2005

70. Santana MJ, Haverman L, Absolom K, et al. Training clinicians in how to use patient-reported outcome measures in routine clinical practice. Qual Life Res. 2015;24(7):1707-1718. doi:10.1007/ s11136-014-0903-5

71. Antunes B, Harding R, Higginson IJ. Implementing patient-reported outcome measures in palliative care clinical practice: a systematic review of facilitators and barriers. Palliat Med. 2014;28(2):158-175. doi: $10.1177 / 0269216313491619$

72. Snyder CF, Jensen R, Courtin SO, Wu AW, Network WfOQAR. PatientViewpoint: a website for patient-reported outcomes assessment. Qual Life Res. 2009;18(7):793-800. doi:10.1007/s11136-009-9497-8
Patient Related Outcome Measures

\section{Publish your work in this journal}

Patient Related Outcome Measures is an international, peer-reviewed, open access journal focusing on treatment outcomes specifically relevant to patients. All aspects of patient care are addressed within the journal and practitioners from all disciplines are invited to submit their work as well as healthcare researchers and patient support groups.
The manuscript management system is completely online and includes a very quick and fair peer-review system. Visit http://www. dovepress.com/testimonials.php to read real quotes from published authors. 